\title{
Investitionsstau und Investitionsbedarf bei den Kommunen
}

Seit Jahren klagen die deutschen Städte und Gemeinden, dass sie nicht genug in den Erhalt und die Erweiterung ihrer Infrastruktur investieren können und dass sich dadurch ein erheblicher Investitionsstau gebildet hat, der gravierende Folgen für die Nutzer der Infrastruktureinrichtungen haben kann. Eine neue Studie des Deutschen Instituts für Urbanistik (Difu) kann diese Klage konkretisieren: Wie groß ist der Investitionsbedarf insgesamt? Welche Summen kommen durch den Investitionsstau zustande? Aber auch zu der in die Zukunft gerichteten Frage nach dem Abbau der Investitionsdefizite wird ein Szenario entwickelt. Ist das neue Konjunkturpaket II der Bundesregierung hier auf dem richtigen Weg? 1

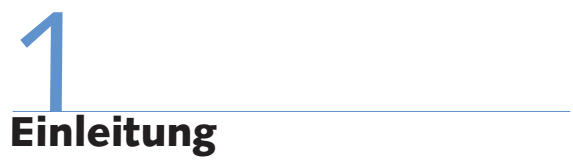

Städte, Gemeinden und Landkreise verfügen über einen großen Bestand an Infrastrukturbauten wie z. B. Straßen, Ver- und Entsorgungseinrichtungen, Verwaltungsgebäude oder Schulen. Eine gute Qualität dieser kommunalen Infrastruktur ist eine wesentliche Voraussetzung für die Lebensqualität der Bürger, für wirtschaftliches Wachstum und politische Stabilität. Diese Infrastruktur dient der Durchführung der kommunalen Tätigkeit selbst, wie bei Verwaltungsgebäuden, überwiegend aber stellt sie eine Vorleistung für die übrigen Wirtschaftsteilnehmer dar, wie beispielsweise die kommunalen Straßen für die Mobilität. Die Kommunen sind für Bau und Betrieb dieser Infrastruktur verantwortlich und haben die Aufgabe, deren Qualität im Rahmen ihrer finanziellen Möglichkeiten zu erhalten und fehlende Strukturen zu ergänzen.

Obwohl sich eine internationale $\mathrm{Fi}$ nanzkrise bereits andeutete, war bis Herbst 2008 die kommunale Finanzsituation insgesamt noch in Ordnung, was sich auch in einem Überschuss der kommunalen Haushalte im Jahre 2008 von fast 10 Mrd. $€$ zeigt. $^{2}$ Das war u. a. die Folge der Unternehmenssteuerreform, mit der es gelang, die Gewerbesteuer am Leben zu erhalten, ja sie sogar zu neuer Blüte zu bringen. Ein beträchtlicher Wermutstropfen war und ist allerdings die Diskrepanz der Ausstattung der Finanzen zwischen den Kommunen, was sich u. a. an dem rapiden Anstieg der Kassenkredite äußerte, die die Defizitkommunen aufnehmen mussten.
Allerdings haben die Kommunen es versäumt, die deutliche Verbesserung ihrer Finanzsituation in wesentlich erhöhte Investitionsvolumina umzusetzen. Durch die abnehmende Investitionstätigkeit entstand ein Investitionsstau, der, wie die Difu-Studie vom Juni 2008 zeigt (Reidenbach et al. 2008), ein beträchtliches Volumen erreicht hat.

Es ist daher eine Ironie der Finanzgeschichte, dass es der größten Finanzkrise der Nachkriegszeit bedarf, um den kommunalen Investitionsstau in erheblichem Umfang abzubauen: Die internationale Finanzkrise und der daraus folgende rapide Konjunkturabschwung haben die Bundesregierung und andere Regierungen zu umfangreichen Maßnahmen veranlasst, mit denen die konjunkturelle Abwärtsbewegung gemäßigt werden soll. Ein erheblicher Teil des Konjunkturpakets II der Bundesregierung besteht in der Stärkung der kommunalen Investitionstätigkeit.

Im Folgenden soll der Investitionsstau und sein möglicher Abbau genauer beschrieben werden. $\mathrm{Zu}$ Beginn steht eine kurze Analyse des kommunalen Vermögens und der Investitionstätigkeit (Abschnitt 2). Danach wird auf die Folgen von vernachlässigter Infrastruktur eingegangen (Abschnitt 3). Abschnitt 4 und 5 beschreiben den kommunalen Investitionsbedarf 2006 bis 2009 und den herrschenden Investitionsstau und entwickeln ein Szenario zu seinem Abbau. Schließlich wird die Verbindung von Investitionsstau und dem Konjunkturpaket II der Bundesregierung hergestellt (Abschnitt 6). Die Schlussbemerkung rundet den Artikel mit Überlegungen zur langfristigen Finanzierung kommunaler Investitionstätigkeit ab (Abschnitt 7).

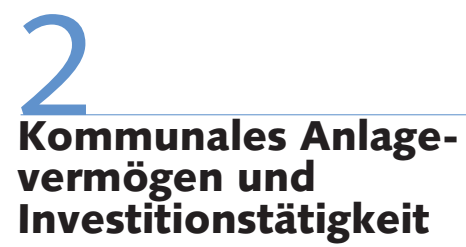

Um die Bedeutung der kommunalen Infrastruktur quantitativ zu beschreiben, ist ein Blick in die Statistik sinnvoll. Im Rahmen der Volkswirtschaftlichen Gesamtrechnungen (VGR) wird auch das Anlagevermögen in der deutschen Wirtschaft berechnet. Auf die Kommunen entfallen bei den Bauten rund $10 \%$ des gesamtwirtschaftlichen Anlagevermögens, bei den Ausrüstungen, das sind Fahrzeuge, Computer etc., sind es nur rund $1 \%$; insgesamt beträgt der kommunale Anteil $9 \%$ (Abbildung 1). In diesen Zahlen ist das Anlagevermögen der kommunalen Unternehmen nicht enthalten. Einschließlich dieser Unternehmen dürfte der Anteil um die $15 \%$ betragen. Schon diese Dimension macht es sinnvoll, auf den Zustand und die Veränderungen des kommunalen Anlagevermögens besonderes Augenmerk zu richten.

\footnotetext{
1 Der Beitrag basiert auf einem Vortrag aus Anlass des WSI-Herbstforums 2008 "Die Rückkehr des Staates", erweitert um einige Aktualisierungen.

2 Siehe die Prognose der kommunalen Spitzenverbände vom 27.1.2009 (http://www.staedtetag.de/ imperia/md/content/pressedien/2009/2.pdf).
}

Michael Reidenbach hat von 1973 bis 2008 am Deutschen Institut für Urbanistik (Difu) Berlin gearbeitet. Arbeitsschwerpunkt: Kommunale Finanzen. e-mail: michael-reidenbach@web.de 
Nur bezogen auf die Kämmereihaushalte ${ }^{3}$ ergibt sich für die kommunale Investitionstätigkeit folgendes Bild:

(1) Die kommunale Investitionstätigkeit ist in den letzen Jahren sowohl in den alten wie in den neuen Bundesländern erheblich zurückgegangen. Dieser Rückgang betraf vor allem Baumaßnahmen (Abbildung 2), weniger den Erwerb beweglicher Güter. Pro Einwohner wurde aber in den neuen Ländern immer noch mehr als in den alten für Baumaßnahmen ausgegeben.

(2) Waren Anfang der 1960er Jahre noch fast ein Drittel der kommunalen Ausgaben in den alten Bundesländern Baumaßnahmen, so ist dieser Anteil inzwischen auf rund $10 \%$ geschrumpft. Auch im internationalen Vergleich (Euro pro Einwohner) rangieren die Investitionen des deutschen Staates am unteren Ende (Diaz Muriel 2007a, 2007b; Reidenbach 2006).

(3) Im Vergleich zu ihrem Anteil am Bruttoanlagevermögen von $9 \%$ waren die Kommunen nur mit etwas über $4 \%$ an den gesamten Bruttoanlageinvestitionen beteiligt (Abbildung 2).

(4) Der Rückgang der Investitionen war sogar so stark, dass die Abschreibungen der Kommunen nach den Berechnungen der VGR höher als die Investitionen ausfielen (Statistisches Bundesamt 2008), das heißt, die Kommunen haben ihr Anlagevermögen abgebaut.

Diese übliche Darstellung der Investitionstätigkeit der Kommunen (Anton/Diemert 2008) vernachlässigt aber die Investitionstätigkeit der kommunalen Unternehmen und Einrichtungen, sodass es zu einer erheblichen Unterschätzung des kommunalen Investitionsvolumens kommt. Am Beispiel des Jahres 2005 lässt sich dieser Sachverhalt verdeutlichen. Danach führten die Kommunen in ihren Kämmereihaushalten rund $20 \mathrm{Mrd}$. $€$ an Sachinvestitionen durch. In den Unternehmen, die ihnen damals ganz oder mindestens zu $50 \%$ gehörten, wurde fast der gleiche Betrag für Sachinvestitionen (genauer: Zugang beim Anlagevermögen) aufgewendet ( $A b$ bildung 3). In den kommunalen Unternehmen werden weitgehend die Investitionen der kommunalen Energieversorgung, der Abwasserbeseitigung oder des kommunalen Krankenhauswesens durchgeführt. $\mathrm{Ob}$

\section{Abb. 1: Anteil der Gemeinden am Bruttoanlagevermögen und an den Bruttoanlageinvestitionen 2005 - in \% -}

Bruttoanlagevermögen

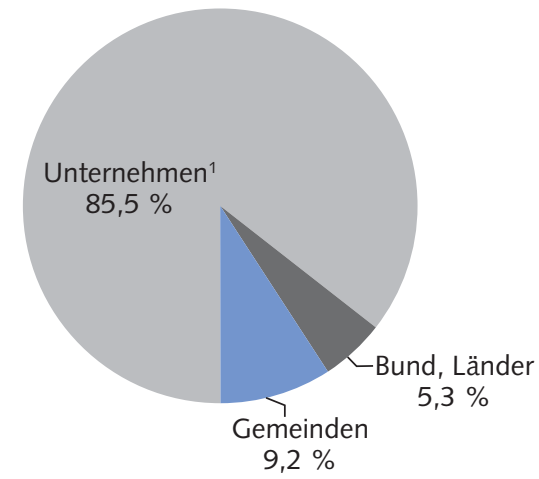

${ }^{1}$ einschließlich Sonstiger und Sozialversicherung. Quelle: Statistisches Bundesamt, VGR.
Bruttoanlageinvestitionen

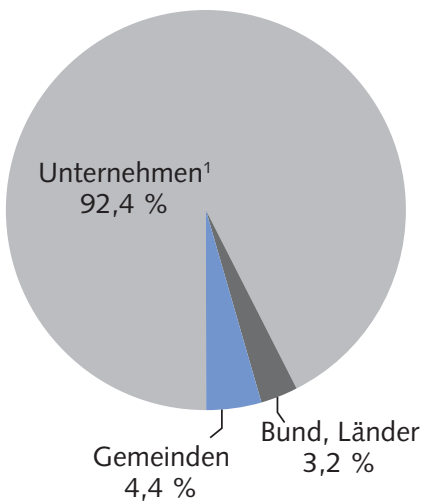

WSI MITTEILUNGEN

\section{Abb. 2: Kommunale Baumaßnahmen 1991 bis 2008}

- in Mrd. Euro in Preisen von 2000 -

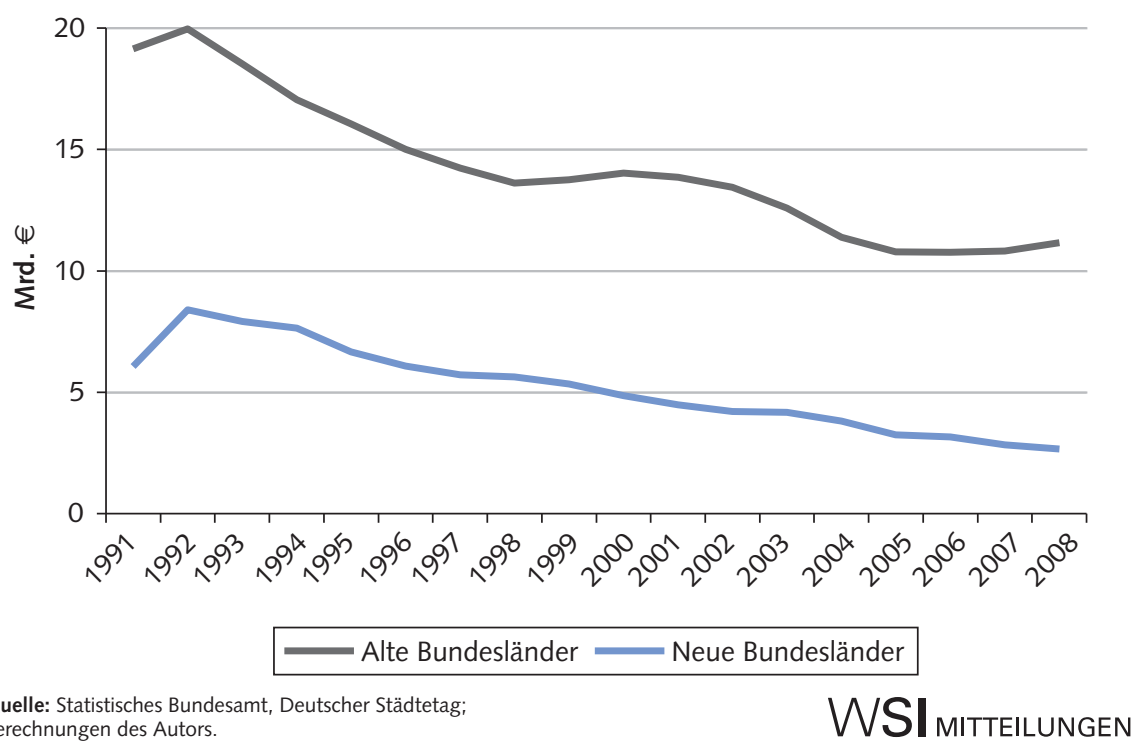

die Investitionen der kommunalen Unternehmen im Zeitverlauf auch zurückgegangen sind, lässt sich mangels statistischer Daten nicht mehr feststellen. ${ }^{4}$

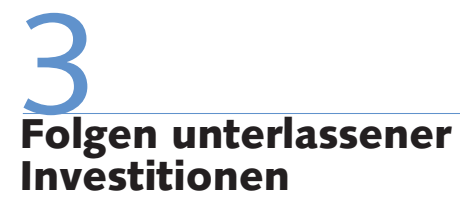

Welches sind die Folgen unterlassener Investitionen im kommunalen Bereich? Diese doch eigentlich wichtige und einfache Frage wird nur relativ selten gestellt und noch seltener beantwortet. Bisher gibt es dazu keine geschlossene Theorie, nur einige Einzelaspekte lassen sich auflisten, die auch häufig nur begrenzt empirisch abgesichert sind:

(1) Langfristig höhere Kosten: Vernachlässigungen bei Unterhalt und Erneuerung der

3 Das sind die Investitionen, die im Rahmen der kommunalen Haushalte durchgeführt werden, im Gegensatz zu den Investitionen, welche kommunale Eigenbetriebe, Zweckverbände und Kapitalgesellschaften tätigen. Diese Abgrenzung entspricht auch dem Gemeindebegriff der VGR.

4 Durch Ausgliederungen aus den Kämmereihaushalten ist das Investitionsvolumen der kommunalen Unternehmen sicherlich vergrößert worden. 


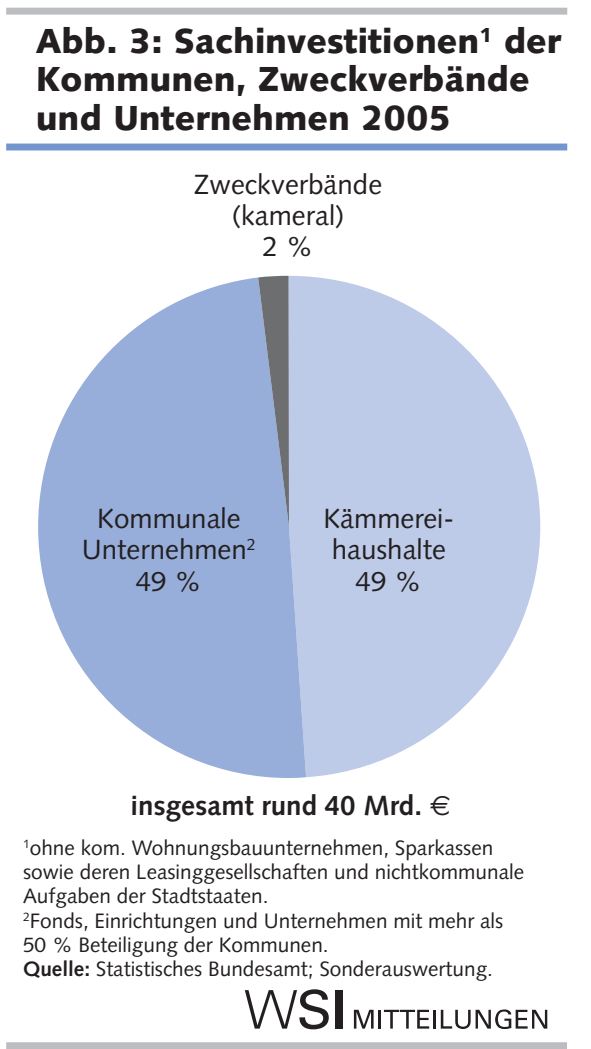

Infrastruktur führen zu stärkerem Verschleiß und wesentlich früherem Ersatzbedarf. So muss beispielsweise die Kommune, unter der Prämisse, dass sie eine entsprechende Straßenqualität anstrebt, später erheblich mehr an Geld aufwenden, wenn ihr nicht genügend Geld zur rechtzeitigen Unterhaltung und Erneuerung der Straßen zur Verfügung steht. Diese Argumentation lässt sich auch auf andere kommunale Anlagen übertragen.

(2) Entgangene Produktion und Wachstum: Ein Defizit an öffentlichem Kapital als Folge der Abnahme der öffentlichen Investitionstätigkeit kann einen Rückgang des Produktivitätswachstums auslösen. Der Zusammenhang gilt für die staatlichen Ebenen wie auch für die kommunale Infrastruktur. Diese Folgewirkung ist von der kurzfristig ausgelegten Stimulation der Wirtschaft durch Konjunkturprogramme zu unterscheiden (vgl. Abschnitt 5), obwohl bei der Ausgestaltung der Konjunkturprogramme der längerfristige Nutzen unbedingt beachtet werden sollte.

(3) Gefährdung der Nutzer: Ein zu geringes Investitionsniveau beispielsweise bei kommunalen Straßen kann für die Nutzer (hier die Verkehrsteilnehmer) erhebliche zusätzliche Gefahren durch nicht gepflegte StraBen und Gehwege mit sich bringen. Auch vernachlässigter Brandschutz, z. B. in Form eines fehlenden zweiten Rettungsweges, kann negative Folgen zeitigen.

(4) Zusätzliche Kosten der Nutzer: Schlechte Straßen führen auch dazu, dass z. B. die Betriebskosten der Autos erheblich ansteigen (Barnes/Langworthy 2003). Auch erhöhte Staukosten können auf fehlende Investitionen in den Ausbau der Straßeninfrastruktur zurückzuführen sein (Keller et al. 2007). Weitere externe Effekte wie Umweltkosten sind dabei noch gar nicht berücksichtigt.

(5) Gebäudequalität und Leistung (outcome): Unterlassene Investitionen wirken sich auch auf die Resultate kommunaler Aufgabenwahrnehmung aus, z. B. auf die Gesundheitsversorgung oder den Bildungserfolg. Die Bedingungen der schulischen Einrichtungen, insbesondere die richtige Raumtemperatur, Beleuchtung und die Akustik, haben einen gewichtigen Einfluss auf die Leistungen der Schüler und die Effektivität der Lehrer (Branham 2002; Lawrence 2003; Sutherland/Hubman 2001).

(6) Umweltfolgen: Undichte Kanäle können beispielsweise eine Exfiltration der Abwässer in den Boden und in das Grundwasser zur Folge haben. So können Schadstoffe, aber auch endokrine Stoffe wie Medikamente, in das Grundwasser gelangen. Ihre Beseitigung ist dann sehr aufwendig. Wird das Grundwasser als Trinkwasser verwendet, entstehen zusätzliche Kosten für die Aufbereitung.

Zwar liegt, wie beschrieben, keine umfassende Darstellung der Folgen unterlassener Modernisierungs- und Sanierungsmaßnahmen vor, doch schon die Beispiele machen deutlich, dass die Auswirkungen erheblich sein können. Es ist klar, dass diese negativen Folgen mit dem Normbild einer guten Qualität der kommunalen Infrastruktur nicht vereinbar sind.

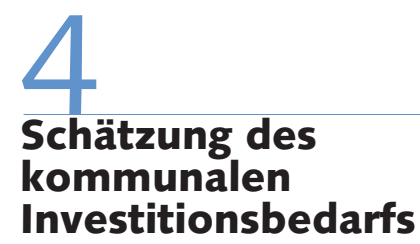

\subsection{METHODISCHE ANMERKUNGEN}

Gegenstand der Schätzung des kommunalen Investitionsbedarfs sind hier von der
Kommune selbst durchgeführte Baumaßnahmen sowie der Erwerb von beweglichen und unbeweglichen Sachen durch sie. Dabei werden auch immaterielle Güter wie Software einbezogen (Abbildung 4).

Die Schätzung ist auf den kommunalen Investitionsbedarf bezogen. Aus diesem Grund werden zum einen die investitionsrelevanten Aufgabenbereiche, die üblicherweise in den Kämmereihaushalten der Städte, Gemeinden und der Gemeindeverbände (Ämter, Landkreise, Bezirke und Landschaftsverbände) eingestellt werden, betrachtet. Einbezogen werden darüber hinaus auch die Investitionsbedarfe der Stadtstaaten, soweit sie als kommunale Aufgabenerfüllung angesehen werden, und der Zweckverbände. Zum anderen wird der Investitionsbedarf der kommunalen Eigenbetriebe sowie derjenigen kommunalen Gesellschaften behandelt, die zu mehr als der Hälfte Eigentum der Kommunen sind.

Die Schätzung des Investitionsbedarfs umfasst die folgenden Infrastrukturbereiche: Trinkwasserleitungen und -anlagen, Straßen, Abwasserleitungen und -anlagen, öffentlicher Personennahverkehr, Schulen, Städtebau und städtebauliche Erneuerung, Verwaltungsgebäude, Krankenhäuser, Sporthallen und Sportstätten sowie sonstige Bereiche und Erwerb von Grundvermögen. Der Bedarf in den sonstigen Bereichen und zum Erwerb von Grundvermögen wird wegen der äußerst heterogenen Zusammensetzung dieser Restkategorie pauschal ermittelt.

Die Bedarfsschätzung wird für die alten Bundesländer (ohne Berlin-West) und die neuen Bundesländer einschließlich Gesamtberlin getrennt vorgenommen. Ermittelt wird der Investitionsbedarf, der im Zeitraum 1.1.2006 bis 31.12.2020 insgesamt gedeckt werden sollte, wenn man den vorhandenen und bis 2020 absehbaren Bedarfsnormen entspräche.

Der gesamte Investitionsbedarf setzt sich aus drei Bedarfskategorien zusammen:

(1) Nachholbedarf entsteht dann, wenn der in der Vergangenheit bis zum Beginn des Betrachtungszeitraumes entstandene Investitionsbedarf nicht ausreichend befriedigt wurde (Investitionsstau).

(2) Erweiterungsbedarf ergibt sich durch die Veränderung der bestimmenden Rahmengrößen, wie z. B. der Bevölkerungs-, Wirtschafts- oder Verkehrsentwicklung, 
aus geänderten strategischen Zielsetzungen oder der Berücksichtigung neuer Normen, wie beispielsweise für die energetische $\mathrm{Sa}$ nierung. Rückbaubedarf ist in diesem Sinne "negativer" Erweiterungsbedarf und geht hinsichtlich des dazu notwendigen Finanzierungsbedarfs in die Schätzung ein.

(3) Ersatzbedarf entsteht, wenn bestehende Bauten und andere Sachanlagen aus technischen oder funktionellen Gründen nicht mehr für nach heutigen Maßstäben notwendige Leistungen tauglich sind und deren Leistung auch in Zukunft benötigt wird.

Zur Schätzung des Erweiterungs- und Nachholbedarfs wird die sogenannte analytische Bedarfsermittlung vorgenommen. Mithilfe dieser Methode wird der Bedarf aus Einzelfaktoren abgeleitet, die seine Höhe und Veränderung im Zeitablauf überwiegend bestimmen, wie die Entwicklung der Bevölkerung in bestimmten Altersgruppen oder die gewerbliche Güterproduktion. Aus der (positiven) Differenz zwischen der im Jahre 2020 angestrebten Versorgung und dem Ende 2005 vorhandenen Bestand ergibt sich das Mengengerüst des Bedarfs. Durch Multiplikation mit entsprechenden Kostenwerten lässt sich daraus der monetäre Bedarf errechnen. Bei dieser Methode spielen also ausschließlich Bedarfskriterien eine Rolle und nicht etwa die kommunale Finanzsituation oder die in der Vergangenheit beobachtbare Über- oder Unterschreitung kommunalpolitisch akzeptierter Versorgungsstandards.

Defizite in der Ausstattung mit kommunaler Infrastruktur treten in der Bundesrepublik in einigen Bereichen nur räumlich begrenzt auf, während andere Räume ausreichend ausgestattet sind oder gar eine Überversorgung aufweisen. Auch wenn im Saldo der Regionen rechnerisch kein Erweiterungsbedarf mehr bestünde, müssen entsprechende Effekte, wie sie u. a. durch Wanderungsbewegungen verstärkt werden, einbezogen werden. Aus diesem Grund wurde die Schätzung für die neuen und die alten Bundesländer getrennt vorgenommen. In Infrastrukturbereichen, in denen starke regionale Ausstattungsunterschiede vorliegen, wurde der räumlich differenzierte Erweiterungs- und Rückbaubedarf pauschal geschätzt.

Zur Ermittlung und Abgrenzung des Bedarfs an Ersatzinvestitionen wurde die sogenannte Kumulationsmethode für die

\section{Abb. 4: Schätzung des kommunalen Investitionsbedarfs}
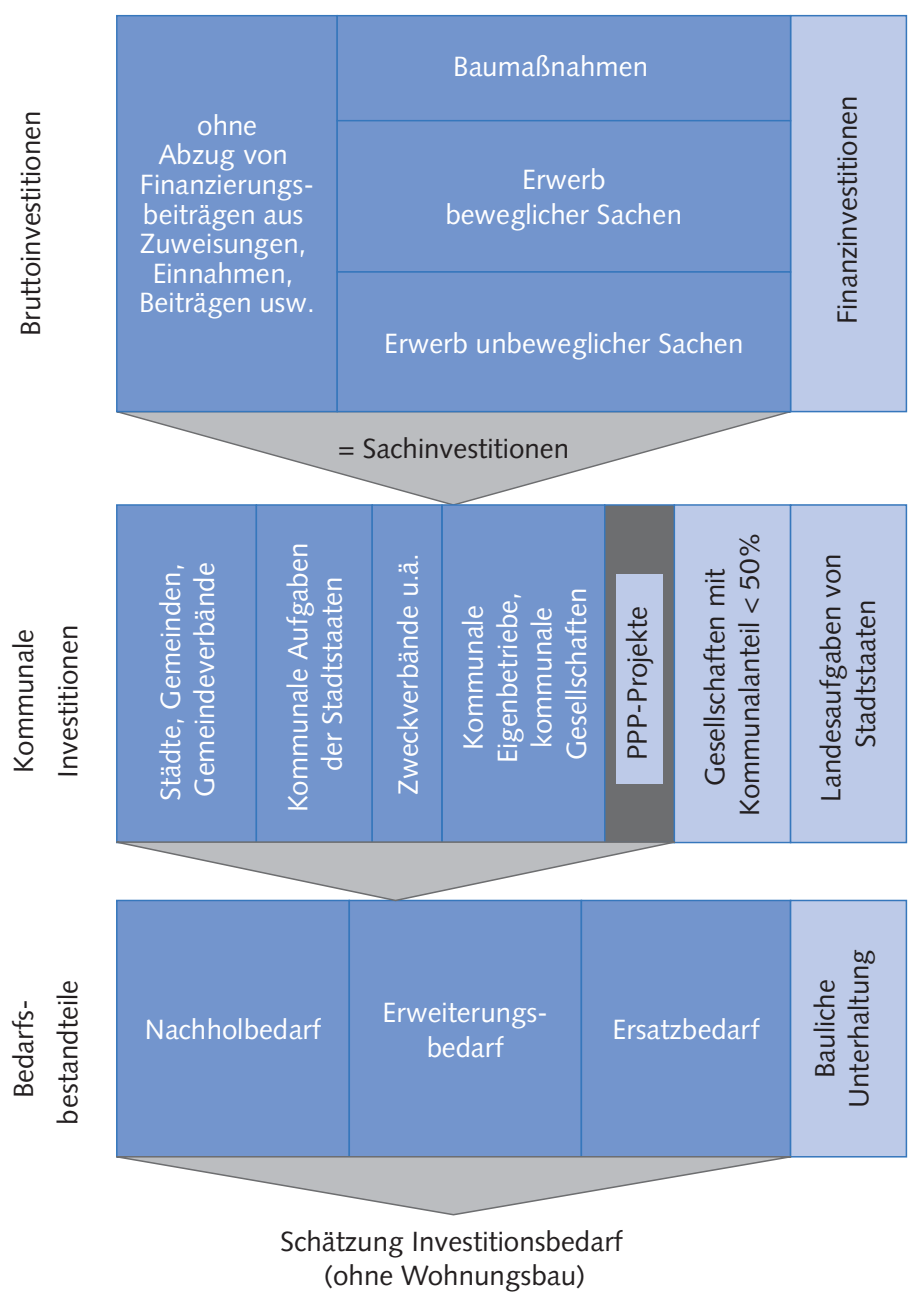

Quelle: Deutsches Institut für Urbanistik.

WSI MITTEILUNGEN

meisten Bereiche angewendet. Dieses Verfahren ist eine in der volkswirtschaftlichen Gesamtrechnung schon lange bekannte und praktizierte Vorgehensweise. Ausschlaggebend für das Ergebnis ist die mittlere Nutzungsdauer, die auf empirischen Untersuchungen fußt. Sie bestimmt, wie schnell oder langsam die in der Vergangenheit getätigten Investitionen aus dem Bestand ausscheiden. Dabei wird anhand der in der Vergangenheit getätigten Investitionen und deren mittlerer Nutzungsdauer der erwartete Abgang getrennt für Bauten und Ausrüstungen ermittelt. In diesen Werten ist eine notwendige Modernisierung z. B. für energetische Gebäudesanierung noch nicht enthalten.

\subsection{RAHMENBEDINGUNGEN FÜR DIE SCHÄTZUNG}

Der zukünftige kommunale Investitionsbedarf steht in engem Zusammenhang mit bestimmten gesamtgesellschaftlichen Entwicklungen in Deutschland. Folgende Grundannahmen wurden getroffen:

(1) Rückläufige Bevölkerungsentwicklung: Nach der Prognose des Bundesamtes für Bauwesen und Raumordnung (BBR) wird sich die Bevölkerung bis zum Jahr 2020 geringfügig verringern und auf 82,1 Mio. Einwohner zurückgehen. Dabei wird für die neuen Bundesländer ein Rückgang von derzeit 16,7 Mio. auf 15,7 Mio. Einwohner angenommen. In den alten Bundesländern wird die Bevölkerung dagegen von derzeit 65,7 Mio. auf 66,4 Mio. Einwohner ansteigen.

(2) Wachsende Anzahl der Haushalte bei abnehmenden Haushaltsgrößen: Aufgrund der zunehmenden Diversifikation und Individualisierung der Lebensstile wird sich der generelle Trend abnehmender Haushaltsgrößen fortsetzen. Dadurch wird die 
Zahl der Haushalte trotz des prognostizierten leichten Rückgangs der Bevölkerung weiter zunehmen.

(3) Wirtschaftswachstum: Für die vorliegende Bedarfsschätzung wird entsprechend den Prognosedaten des Instituts für Wirtschaftsforschung Halle (IWH) für die alten Bundesländer von einem durchschnittlichen Wachstum von 1,7\% und für die neuen Bundesländer von 1,6 \% ausgegangen. ${ }^{5}$

(4) Verkehrsentwicklung: Es wird mit einem noch leicht steigenden Pkw-Bestand gerechnet. In dynamischen Regionen wird von noch leicht wachsenden, in Regionen mit Bevölkerungsabnahme sinkenden Verkehrsleistungen verbunden mit einer Abflachung der Verkehrsspitzen ausgegangen. Der die Kommunalstraßen in besonderem Maße belastende innerörtliche Güterumschlag verlagert sich weiter an den Stadtrand und auf Landes- und Bundesstraßen. In den Kernstädten und in den Stadt-Umland-Relationen bleibt der öffentliche Personennahverkehr stark und bedeutsam, in kleinen Städten und außerhalb der Städte ist im klassischen Busverkehr mit deutlichen Einbrüchen zu rechnen.

Neben der Bevölkerungs-, Wirtschaftsund Verkehrsentwicklung hängt der zukünftige Investitionsbedarf auch vom angestrebten Qualitätsmaßstab der kommunalen Leistungserbringung ab. Dieser wird in hohem Maße politisch bestimmt und fließt zum Teil in entsprechende gesetzliche Regelungen mit ein. Bestimmte Tendenzen in diesem Bereich, die absehbar sind bzw. bereits ihren Niederschlag in Gesetzesform gefunden haben, werden im Rahmen der Schätzung benannt, konnten aber nur in wenigen Teilbereichen quantitativ untersetzt werden. Die folgenden Aspekte sind dabei von besonderer Bedeutung:

(1) Sicherheit: Die zukünftige Investitionskostenentwicklung in diesem Bereich wird wesentlich davon bestimmt werden, wie sich Bedrohungs- und Gefährdungslagen in unterschiedlichen städtischen Räumen entwickeln und wie Sicherheits- und Schutzbedürfnisse für spezifische kommunale Einrichtungen eingeschätzt werden.

(2) Klimawandel und Klimaschutz: Sie äußern sich vor allem in den drei investitionsrelevanten Tatbeständen Beseitigung der Schäden durch Klimafolgen, Katastrophenschutz und Klimaschutz. Eine Schätzung des Bedarfs wurde dabei für den Bereich Klimaschutz und hier nur für die energetische Sanierung der kommunalen Gebäude vorgenommen.

\section{5 \\ Ergebnisse der Investitions- bedarfsschätzung}

Für die Jahre 2006 bis 2020 ergibt die Schätzung einen kommunalen Investitionsbedarf von $704 \mathrm{Mrd}$. $€$ in Preisen von 2000. Das entspricht einem Investitionsbedarf von knapp 47 Mrd. $€$ pro Jahr. Pro Einwohner fallen in den alten Bundesländern für die 15 Jahre 2006 bis 2020 jährlich $755 €$ an, in den neuen Bundesländern und Berlin sind es $858 €$, also rund $14 \%$ mehr.

Die größte Bedarfskategorie ist der Ersatzbedarf mit $59 \%$. Der Erweiterungsbedarf entspricht einem Anteil von $31 \%$ und der Nachholbedarf macht $10 \%$ des Investitionsbedarfs aus (Abbildung 5). Das tatsächliche Investitionsniveau des Jahres 2005 liegt für die hier einbezogenen Bereiche insgesamt bei ca. $40 \mathrm{Mrd}$. $€$. Die Investitionen werden, wie beschrieben, jeweils etwa zur Hälfte durch die Kommunen selbst und durch die kommunalen Unternehmen aufgebracht. Die Gegenüberstellung zeigt, dass mit einem Investitionsniveau in dieser Höhe zukünftig zumindest der jährliche Erweiterungs- und Ersatzbedarf weitestgehend abgedeckt wären. Der

\section{Abb. 5: Kommunaler Investi- tionsbedarf 2006 bis 2020}

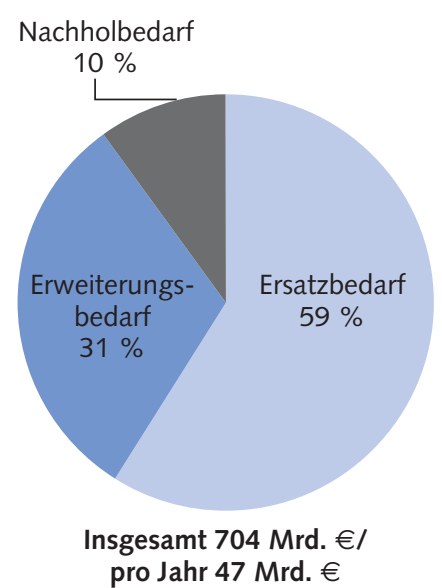

Quelle: Deutsches Institut für Urbanistik.

WSI MITTELLUNGEN
Investitionsstau als die Differenz zwischen Bedarf und tatsächlichen Investitionen beläuft sich auf etwas über $70 \mathrm{Mrd}$. $€$, das entspricht größenordnungsmäßig dem Nachholbedarf.

Insgesamt zeigt sich, dass im Vergleich der einzelnen untersuchten Infrastrukturbereiche der größte Investitionsbedarf bis 2020 in den Bereichen Straßenbau (162 Mrd. €), Schulen (73 Mrd. €) und kommunale Abwasseranlagen (58 Mrd. €) besteht (Tabelle 1). ${ }^{6}$

Der Schwerpunkt des Investitionsbedarfs im Bereich des Straßenbaus liegt mit 71 Mrd. $€$ beim Ersatzbedarf für vorhandene Straßen, Brücken und Tunnelanlagen. Der Erweiterungsbedarf im Straßenbau (24 Mrd. €) konzentriert sich weitgehend auf Erschließungsmaßnahmen. Nur vereinzelt gibt es noch Bedarf an neuen kommunalen Hauptverkehrsstraßen. Nachholbedarf besteht vor allem in der Umgestaltung von Kommunalstraßen (31 Mrd. €) mit einem Schwerpunkt in den neuen Bundesländern. Neben dem unmittelbaren Bau von Straßen werden für Einrichtungen für den Geh- und Fahrradverkehr, Verkehrsinformations- und Steuerungssysteme, Lärmschutzeinrichtungen und Parkierungsanlagen zusammen $36 \mathrm{Mrd}$ € veranschlagt.

Im Bereich der kommunalen Schulen besteht der größte Investitionsbedarf bei Erweiterungsmaßnahmen. Die Anpassung der Kapazität macht etwa $48 \%$ des ermittelten Investitionsbedarfs aus. Die Grundlage dafür ist jedoch nicht die Entwicklung der Schülerzahlen im Betrachtungszeitraum. Die Auslöser für den Investitionsbedarf sind vielmehr in sich ändernden räumlichen und technischen Anforderungen an Schulgebäude, wie z. B. Maßnahmen zur energetischen Sanierung, Erweiterungen aufgrund veränderter Schulkonzepte (G8), zu sehen. Etwa ein Fünftel des Erweiterungsbedarfs betrifft die Erweiterung der Ausrüstung mit Computern.

Den Schwerpunkt des Investitionsbedarfs der kommunalen Abwasserbeseitigung bilden mit Abstand die Ersatzinvestitionen in Höhe von knapp $44 \mathrm{Mrd}$. $€$. Da-

5 Das war möglicherweise zu optimistisch, aber der Einfluss der Wirtschaftsentwicklung auf den kommunalen Investitionsbedarf ist nur in einigen Bereichen wie z. B. dem Güterverkehr erheblich.

6 Die sonstigen Bereiche teilen sich auf in solche, die im Haushalt geführt werden (91 Mrd. €), die Energieversorgung mit $60 \mathrm{Mrd} . €$ und die Investitionen der übrigen kommunalen Unternehmen (58 Mrd. €). 
von entfallen ca. die Hälfte auf Maßnahmen im Bereich des Kanalnetzes und jeweils ein Viertel auf den baulichen Teil der Kläranlagen sowie die Maschinen- und Elektrotechnik.

Eine vergleichende Betrachtung der alten und neuen Bundesländer ergibt ein grundsätzlich ähnliches Bild bei den Investitionsschwerpunkten. Allerdings wird die herausragende Bedeutung der Investitionen in die kommunalen Straßen in den neuen Bundesländern noch deutlicher. Der Investitionsbedarf bis 2020 beträgt in diesem Bereich 43,3 Mrd. € und macht damit $27 \%$ des gesamten Bedarfs aus (in den alten Bundesländern $22 \%$ ).

Klar erkennbar wird auch der immer noch vorhandene Nachholbedarf in den neuen Bundesländern. Dort ergeben sich in den differenziert geschätzten Bereichen insgesamt $24 \%$ des Bedarfes aus dem Investitionsrückstand. In den alten Bundesländern entspricht dieser Anteil nur $6 \%$. Der größte Nachholbedarf besteht bei den kommunalen Straßen mit 19,8 Mrd. € in den neuen Bundesländern (entspricht $46 \%$ des Bedarfs im Bereich Straßen) und 11,1 Mrd. $€$ in den alten Bundesländern (entspricht dort $9 \%$ des Bedarfs).

Während der Anteil der Ersatzinvestitionen in den alten Bundesländern fast zwei Drittel des Investitionsbedarfs ausmacht, beträgt deren Anteil in den neuen Bundesländern nur $47 \%$. Eine Ursache dafür ist in den umfangreichen Investitionen nach der Wiedervereinigung zu sehen. Die langlebigen Anlagegüter wie Abwassernetze befinden sich noch am Beginn ihrer Nutzungszeit.

Der Erweiterungsbedarf ist in den Bereichen Abwasser, Schulen und Straßen von besonderer Bedeutung. Bei der Abwasserbeseitigung ergibt sich dies in erster Linie aus der noch andauernden Erhöhung des Anschlussgrades in den neuen Bundesländern. Der Erweiterungsbedarf beläuft sich dort auf 5,1 Mrd. $€$. Der Erweiterungsbedarf im Schulbereich beträgt in den alten Bundesländern 30,0 Mrd. € und in den neuen 4,8 Mrd. $€$. Der Erweiterungsbedarf bei den kommunalen Straßen besteht mit 43,8 Mrd. $€$ in den alten und 12,4 Mrd. $€$ in den neuen Bundesländern insbesondere aus dem Ausbau des Straßennetzes im Zusammenhang mit der anhaltenden privaten und gewerblichen Neubautätigkeit.

Gegenüber der Schätzung des kommunalen Investitionsbedarfs 2000 bis 2009

Tabelle 1: Investitionsbedarf der Kommunen und ihrer Unternehmen 2006 bis 2020

\begin{tabular}{|c|c|c|c|c|c|c|c|c|c|}
\hline & \multirow[t]{2}{*}{ Schätzbereiche } & \multicolumn{3}{|c|}{ Alte Bundesländer } & \multicolumn{3}{|c|}{$\begin{array}{l}\text { Neue Bundesländer } \\
\text { und Berlin }\end{array}$} & \multicolumn{2}{|c|}{$\begin{array}{l}\text { Deutschland } \\
\text { insgesamt }\end{array}$} \\
\hline & & $\begin{array}{l}\text { in Mrd. } \\
\text { Euro }\end{array}$ & in $\%$ & $\begin{array}{l}\text { in Euro/ } \\
\text { Einw. }\end{array}$ & $\begin{array}{l}\text { in Mrd. } \\
\text { Euro }\end{array}$ & in $\%$ & $\begin{array}{l}\text { in Euro/ } \\
\text { Einw. }\end{array}$ & $\begin{array}{l}\text { in Mrd. } \\
\text { Euro }\end{array}$ & in $\%$ \\
\hline \multirow{9}{*}{ 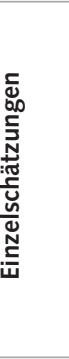 } & Trinkwasser & 21,5 & 3,9 & 327 & 7,5 & 4,7 & 447 & 29,0 & 4,1 \\
\hline & Abwasser & 45,8 & 8,4 & 697 & 12,4 & 7,8 & 739 & 58,2 & 8,3 \\
\hline & Verwaltungsgebäude & 16,9 & 3,1 & 257 & 2,9 & 1,8 & 173 & 19,8 & 2,8 \\
\hline & Krankenhäuser & 23,7 & 4,3 & 361 & 7,2 & 4,5 & 429 & 30,9 & 4,4 \\
\hline & $\begin{array}{l}\text { Schulen } \\
\text { (ohne Sporthallen) }\end{array}$ & 61,1 & 11,2 & 930 & 11,9 & 7,5 & 709 & 73,0 & 10,4 \\
\hline & Sportstätten & 27,1 & 5,0 & 413 & 8,1 & 5,1 & 483 & 35,2 & 5,0 \\
\hline & Straßen & 118,3 & 21,7 & 1.801 & 43,3 & 27,3 & 2.580 & 161,6 & 22,9 \\
\hline & ÖPNV & 30,4 & 5,6 & 463 & 8 & 5,1 & 477 & 38,4 & 5,5 \\
\hline & Städtebau & 6,3 & 1,2 & 96 & 3,8 & 2,4 & 226 & 10,1 & 1,4 \\
\hline \multirow{5}{*}{ 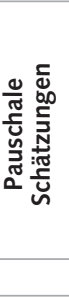 } & $\begin{array}{l}\text { Sonstige Bereiche } \\
\text { im kom. Haushalt }\end{array}$ & 63,8 & 11,7 & 971 & 27,5 & 17,4 & 1.639 & 91,3 & 13,0 \\
\hline & $\begin{array}{l}\text { Kom. } \\
\text { Energieversorgung }\end{array}$ & 51,8 & 9,5 & 789 & 8,1 & 5,1 & 483 & 59,9 & 8,5 \\
\hline & $\begin{array}{l}\text { Restliche kom. } \\
\text { Unternehmen }\end{array}$ & 44,4 & 8,1 & 676 & 12,9 & 8,1 & 769 & 57,3 & 8,1 \\
\hline & $\begin{array}{l}\text { Erwerb von } \\
\text { Grundvermögen }\end{array}$ & 34,7 & 6,4 & 528 & 4,8 & 3,0 & 286 & 39,5 & 5,6 \\
\hline & Summe /Mittelwert & 545,8 & 100,0 & 8.309 & 158,4 & 100,0 & 9.439 & 704,2 & 100,0 \\
\hline
\end{tabular}

gibt es verschiedene Abweichungen bei der methodischen Herangehensweise. So wurde der Untersuchungsgegenstand stärker auf die kommunalen Kernaufgaben fokussiert. Aus diesem Grund wurden die Bereiche kommunaler Wohnungsbau und kommunale Finanzinvestitionen nicht mehr einbezogen. Darüber hinaus konnte auf einer gegenüber der vorherigen Schätzung verbesserten Datenlage aufgebaut werden. Insbesondere für die neuen Bundesländer konnten pauschale Ansätze durch differenziertere Schätzungen ergänzt bzw. ersetzt werden. Die genannten Anpassungen wurden im Interesse einer höheren Belastbarkeit der Ergebnisse dieser Studie vorgenommen, auch wenn dadurch ein direkter Vergleich der Ergebnisse beider Schätzungen nicht mehr aussagekräftig ist.

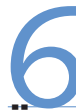 Überlegungen zum Abbau des Investitionsstaus}

\subsection{SZENARIO}

Angesichts des anhaltend hohen kommunalen Investitionsstaus stellt sich für die Kommunen die Frage, wie sie diesen Finanzbedarf in den kommenden Jahren decken können.

Mögliche Lösungsansätze werden in einer verstärkten strategischen Ausrichtung des Investitionsmanagements unter Anwendung des Lebenszyklusansatzes und des Einsatzes kostenminimierender Unterhaltungsstrategien gesehen. Darüber hinaus gibt es eine Vielzahl von Stellschrauben, mit denen die Finanzierung bzw. Finanzierbarkeit von Investitionen beeinflusst werden kann. So stehen den Kommunen ganz unterschiedliche Möglichkeiten zur Beschaffung zusätzlicher Einnahmen zur Verfügung, die der Finanzierung von Investitionen dienen können. Von besonderer Bedeutung sind dabei die Erhebung von Gebühren und Beiträgen sowie der Einsatz von Fremdfinanzierungsmodellen wie beispielsweise dem Contracting, welches unter Umständen ganz ohne Kapitaleinsatz durch die Kommunen auskommen kann.

Die Beteiligung privater Partner im Rahmen von Public-Private-Partnerships (PPP) stellt einen integrierten Strategieund Finanzierungsansatz in ausgewählten Infrastrukturbereichen, etwa bei Schulen oder im Bereich Sport/Freizeit/Tourismus, dar. Dieser Ansatz hat sich als wichtige Handlungsoption erwiesen, wenn es darum geht, die kommunale Infrastruktur effizient zu betreiben. Der anhaltende Bedarf an kommunaler Investitionstätigkeit macht eine Prüfung weiterer Investitionsbereiche nach ihrer PPP-Geeignetheit notwendig. Ein entsprechendes Prüfraster wurde im Zusammenhang mit der Investitionsbedarfsschätzung erarbeitet. 


\section{Tabelle 2: Finanzierungsquellen zum Abbau des kommunalen Investitionsstaus (Szenario 2009-2020)}

\begin{tabular}{lr}
\hline & Mrd. Euro \\
\hline Investitionsstau (einschl. 5 Mrd. € 2006-2008) & 75,0 \\
Finanziert durch folgende Maßnahmen & \\
Strategisches Investitionsmanagement (ohne PPP), & 30,6 \\
davon: & 28,4 \\
$\quad$ Anwendung des Lebenszyklusansatzes, Realisierung von Größenvorteilen \\
$\quad$ und leistungswirtschaftlichen Vorteilen, kostenoptimierende Instandhaltungs- \\
$\quad$ und Modernisierungsprogramme & 2,2 \\
Flexibilität der Infrastruktureinrichtungen & 15,0 \\
PPP-Projekte & 29,4 \\
Einsatz von Finanzierungsinstrumenten, davon: & 7,1 \\
$\quad$ Entgelte & 4,5 \\
Contracting & 5,3 \\
Zusätzliche Steuern, Verkäufe und Kredite & 12,5 \\
Zuweisungen, Förderprogramme usw. von Bund, Ländern und EU & \\
\hline & \\
Quelle: Deutsches Institut für Urbanistik. & MITTEILUNGEN
\end{tabular}

Der Abbau des Investitionsrückstands und die Deckung des kommunalen Investitionsbedarfs bis 2020 sind machbar, wenn alle Optionen eines strategischen Investitionsmanagements, von integrierten und verträglichen Finanzierungslösungen sowie von Effizienzgewinnen etwa durch PPP-Projekte ausgeschöpft werden und wenn gleichzeitig Bund, Länder und Kommunen im Rahmen ihrer Möglichkeiten eine politische Priorisierung eines entsprechenden Investitionsprogramms vornehmen.

Politisches und programmatisches Ziel müsste es dabei sein, diesen Investitionsrückstand nicht gleichmäßig verteilt bis zum Jahr 2020 abzutragen, sondern - zur möglichst schnellen Beseitigung der Folgen unterlassener Investitionen - in einer starken und konzertierten Aktion in den Jahren 2009 bis 2015 die Investitionsanstrengungen zu forcieren.

Der Abbau des Investitionsrückstands und die Deckung des Investitionsbedarfs bis zum Jahr 2020 könnten aus den in $\mathrm{Ta}$ belle 2 dargestellten Quellen gespeist werden. Die Zahlen sind einerseits aus Studien abgeleitet, aus denen sich mögliche Einsparpotenziale ergeben, und sind andererseits Setzungen, die vor dem Hintergrund der öffentlichen Finanzsituation bei politischem Willen realistisch darstellbar wären.

Es muss an dieser Stelle allerdings betont werden, dass es sich bei der Darstellung um ein Szenario handelt, das neue strategische Ausrichtungen und politische Priorisierungen auf allen staatlichen Ebenen voraussetzt. Es wird keine Aussage darüber getroffen, als wie wahrscheinlich das Eintreten dieses Szenarios einzuschätzen ist.
Der Einsatz von zusätzlichen Finanzmitteln als Folge der politischen Willensbekundung müsste im Schwerpunkt in den Jahren 2009 bis 2012 erfolgen. Die positiven Effekte durch strategisches Investitionsmanagement, den Einsatz intelligenter Finanzierungsinstrumente und PPPProjekte werden stärker erst in den Jahren nach 2012 greifen, dann aber besonders nachhaltige Wirkung weit nach dem Jahr 2020 erzeugen.

\subsection{INVESTITIONSBEDARF UND KONJUNKTURPAKET}

Seit der Veröffentlichung der Studie über den kommunalen Investitionsbedarf im Juni 2008 sind als Folge der Finanzmarktund Wirtschaftskrise von der Bundesregierung unter Mitfinanzierung von Ländern und Kommunen zwei Konjunkturpakete aufgelegt worden. Das erste Paket (Beschäftigungssicherung durch Wachstumsstärkung) konzentrierte sich aus Sicht der Kommunen im Wesentlichen auf verbilligte Kredite für energetische Maßnahmen, die Kommunen müssen aber die Steuererleichterungen dieses Programms in etwa gleicher Höhe mitfinanzieren. ${ }^{7}$ Das Konjunkturprogramm II (Gesetz zur Sicherung von Beschäftigung und Stabilität in Deutschland) enthält in seinem Artikel 7 das Gesetz zur Umsetzung von Zukunftsinvestitionen der Kommunen und Länder (Zukunftsinvestitionsgesetz), das im Hinblick auf die Deckung des Investitionsbedarfs wesentlich interessanter ist.

Geht man von dem Gesetzentwurf und der dazu gehörenden Verwaltungsvereinbarung aus, ${ }^{8}$ dann werden von den 49,8 Mrd. € des Konjunkturprogramms II
17,3 Mrd. € für staatliche Infrastrukturinvestitionen verwendet. Von diesen sind 4 Mrd. € Maßnahmen des Bundes (hauptsächlich Straßenbau), vom Rest (13,3 Mrd. $€)$ sollen mindestens $70 \%$ auf kommunalbezogene Investitionen (= 9,6 Mrd. €) entfallen, zu denen auch Mittel für private Schulen sowie nichtkommunale Kindergärten zählen (Tabelle 3 für Bayern). Von diesen 13,3 Mrd. $€$ bringt der Bund aus einem speziellen Fonds $10 \mathrm{Mrd}$. $€$ auf, der Rest ist gemeinsam vom jeweiligen Land und seinen Kommunen zu finanzieren; eine Vorgabe des Bundes über den jeweiligen Anteil gibt es nicht. Von den 13,3 Mrd. $€$ sind $65 \%$ für Bildung und $35 \%$ für Infrastruktur zu verwenden.

Wie ist dieses Konjunkturpaket II unter dem Gesichtspunkt Deckung des kommunalen Investitionsstaus zu beurteilen? Dazu gibt es keine eindeutige Antwort:

(1) Das Zukunftsinvestitionsgesetz soll das Herzstück des Konjunkturpakets II sein. Der Betrag von 9,6 Mrd. $€$ ist ein erfreulicher und unerwarteter Geldsegen für die Kommunen. Sogar der Sachverständigenrat, der sonst solchen Programmen sehr skeptisch gegenüberstand, hielt ein solches Programm zum Abbau des kommunalen Investitionsstaus für notwendig (SVR 2008). Der an die Länder und die Kommunen gehende Betrag macht aber nur $27 \%$ der Gesamtsumme des Konjunkturpakets II aus.

(2) Die Verteilung der Mittel des Bundes an die Länder (und die Kommunen) wird nach einem Mix von Schlüsseln berechnet. Die dadurch erzeugte Verteilung der Mittel führt dazu, dass die Länder und Kommunen in den neuen Bundesländern pro Einwohner $140 €$, in den alten Bundesländern $116 €$ erhalten. Diese Differenz reflektiert auch den erwähnten unterschiedlichen kommunalen Investitionsbedarf.

\footnotetext{
Siehe Entwurf eines Gesetzes zur Umsetzung steuerrechtlicher Regelungen des Maßnahmenpakets Beschäftigungssicherung durch Wachstumsstärkung, Bundestags-Drucksache 16/10930 vom 13.11.2008.

8 Entwurf eines Gesetzes zur Sicherung von Beschäftigung und Stabilität in Deutschland (BTDrucksache 16/11740 vom 27.1.2009), die Änderungen durch den Haushaltsausschuss (BT-Drucksachen 16/11801 und 16/11825), der Beschluss des Bundesrates (120/09 vom 20.2.2009) und die Verwaltungsvereinbarung zur Durchführung des Gesetzes (Entwurf Stand 27.1.2009).
} 
(3) Zwischen Ländern und Kommunen ist nach der Verwaltungsvereinbarung eigentlich eine 30:70-Verteilung vorgesehen. Das Land Nordrhein-Westfalen erhöht den kommunalen Anteil, andere Länder tun sich damit schwer und versuchen, Landesaufgaben in kommunale Aufgaben umzudeklarieren, um so selbst an die Mittel zu kommen. Hier wie an anderen Stellen kommt die Problematik der Mittelflüsse vom Bund an die Kommunen zum Tragen, da die Länder zwischengeschaltet sind und Größe und Struktur des Mittelflusses beeinflussen wollen.

(4) Bei der Verteilung der Mittel zwischen den Kommunen zeichnen sich zwei Lösungswege ab: Zum einen eine pauschale Verteilung nach Schlüsseln des kommunalen Finanzausgleichs, wie sie in NRW erfolgt. ${ }^{9}$ Die finanzschwachen Kommunen werden besonders dotiert. ${ }^{10}$ Alle Kommunen werden beteiligt, eine Ausrichtung am konkreten Bedarf erfolgt nicht. Es wird unterstellt, dass er überall vorhanden ist. Der Vorteil ist die zügige Verteilung der Mittel, die einzelne Gemeinde muss selbst auf die Einhaltung der Vorschriften achten. Zum anderen ist wie in Bayern eine Verteilung der Mittel nach beantragten Projekten vorgesehen, wo letztlich die Landesbürokratie und die Landespolitiker über die Verteilung entscheiden. ${ }^{11}$ Dies kann zu einer besseren Kontrolle der Mittelverwendung führen, ist aber schwerfälliger und schränkt die kommunale Selbstbestimmung ein.

(5) Im Falle der Projektfinanzierung sollen die Kommunen also auch einen, wenn auch kleinen, Eigenanteil erbringen. Zum einen sollte der Eigenanteil die Bereitschaft fördern, mit den Zuweisungsmitteln sorgfältig umzugehen, zum anderen ist es das Ziel des Bundes, vor allem die energetische Sanierung der kommunalen Gebäude voranzubringen. Eine solche Sanierung führt in der Regel zu erheblichen Einsparungen an Heizmitteln, aus denen sich der kommunale Eigenanteil meist gut finanzieren lässt.

(6) Hauptproblem mit Blick auf den Investitionsstau ist die Ausklammerung wichtiger kommunaler Bereiche, denn die Verwendungszwecke sind per Gesetz vorgegeben. So wird der Bereich Abwasser- und Abfallbeseitigung nicht berücksichtigt, weil er voll durch Gebühren gedeckt wird. Beim kommunalen Straßenbau sind nur Lärm-

Tabelle 3: Verteilung der Mittel des Konjunkturpakets II 2009 bis 2011 in Bayern

\begin{tabular}{|c|c|c|c|c|}
\hline \multirow[t]{2}{*}{ Maßnahme } & \multirow{2}{*}{$\begin{array}{c}\text { Volumen } \\
\text { in Mio. } \\
\text { Euro }\end{array}$} & \multicolumn{3}{|c|}{ Kofinanzierungssätze } \\
\hline & & $\begin{array}{l}\text { Bund } \\
\text { in } \%\end{array}$ & $\begin{array}{l}\text { Land } \\
\text { in } \%\end{array}$ & $\begin{array}{l}\text { Kommunen } \\
\text { in \% }\end{array}$ \\
\hline $\begin{array}{l}\text { § } 3 \text { Abs. } 1 \text { Nr.1 (Kommunale Bildung) } \\
\text { darunter }\end{array}$ & 906,8 & & & \\
\hline Energetische Sanierung Schulen & 573,3 & 75,0 & 12,5 & 12,5 \\
\hline $\begin{array}{l}\text { Energetische Sanierung sonstige } \\
\text { Bildungseinrichtungen }\end{array}$ & 179,6 & 75,0 & 12,5 & 12,5 \\
\hline $\begin{array}{l}\text { Energetische Sanierung von Privat-/ } \\
\text { und kirchlichen Schulen }\end{array}$ & 50,0 & 75,0 & 25,0 & 0,0 \\
\hline Übrige Maßnahmen & 103,8 & & & \\
\hline $\begin{array}{l}\text { § } 3 \text { Abs. } 1 \text { Nr.2 (Kommunale Infrastruktur) } \\
\text { darunter: }\end{array}$ & 488,3 & & & \\
\hline Krankenhausbaumaßnahmen & 40,0 & 75,0 & 12,5 & 12,5 \\
\hline Energieeinsparung Krankenhäuser & 70,0 & 75,0 & 12,5 & 2,5 \\
\hline Energetische Sanierung sonstiger Infrastruktur & 89,7 & 75,0 & 12,5 & 12,5 \\
\hline Städtebau & 30,0 & 60,0 & 0,0 & 40,0 \\
\hline Dorferneuerung & 30,0 & 60,0 & 0,0 & 40,0 \\
\hline Lärmsanierung Kommunalstraßen & 45,0 & 75,0 & 12,5 & 12,5 \\
\hline Breitband & 50,0 & 37,5 & 12,5 & 50,0 \\
\hline Übrige Maßnahmen & 133,6 & & & \\
\hline
\end{tabular}

schutzmaßnahmen an kommunalen Straßen zuwendungsfähig, der ÖPNV ist ebenfalls ausgeklammert. Damit kommt nur ein kleiner Teil der möglichen Investitionsbereiche für Zuweisungen des Staates in Betracht.

(7) Die Mittel sollen zügig abgerufen werden, ${ }^{12}$ mindestens die Hälfte schon 2009. ${ }^{13}$ Auch diese Regelung ist für eine schnelle Wirkung des Konjunkturpakets unerlässlich, dürfte aber bei strikter Einhaltung zu Hektik am Jahresende und Verzicht auf an sich notwendige Kontrollen führen. Projekte, die jetzt noch nicht fertig sind (shovel-ready), dürften tendenziell für einen längeren Zeitraum verschoben werden. Das dürfte auch für PPP-Projekte gelten, deren private Seiten ohnehin von der Finanzkrise betroffen sind.

(8) Die Zusätzlichkeit der Ausgaben ist erforderlich, damit entsprechende Multiplikatorwirkungen entstehen und damit der Investitionsstau auch abgebaut wird. Gleichwohl ist die praktische Umsetzung dieses Gebotes sehr schwierig. Von den Ländern wird bereits eine Änderung der Bestimmung der Zusätzlichkeit angemahnt (BR-Drucksache 120/09 vom 20.2.2009).

(9) Die Klausel, dass Investitionen nur zulässig sind, wenn deren längerfristige Nutzung auch unter Berücksichtigung der absehbaren demografischen Veränderungen vorgesehen ist ( $\$ 4$ Abs 3 Entwurf des
Zukunftsinvestitionsgesetzes), erscheint gerade im Hinblick auf den Planungshorizont 2020 bei der Ermittlung des Investitionsbedarfs sehr sinnvoll zu sein. Allerdings wird sich wahrscheinlich zeigen, dass viele Kommunen keine derartige langfristige, an sich notwendige Schulentwicklungsplanung aufweisen.

Insgesamt gesehen stellt das Konjunkturpaket einen wichtigen Schritt zum Abbau des kommunalen Investitionsstaus dar, der ruhig etwa höher hätte ausfallen können. Andererseits muss die Bauverwaltung ein gegenüber früheren Jahren erheblich ge-

\footnotetext{
9 Die Schlüssel sind Einwohnerzahl, Fläche, Finanzkraft und Schülerzahl. (http://www.nrw.de/Presseservice/archive/presse2009/01_2009/Gemeinsame_Erkl_rung_MP_mit_kommSpitzen.pdf).

10 Die Verteilung der Mittel wurde in einer Modellrechnung offen gelegt (http://www.im.nrw.de/ bue/doks/0902modellrechnungkp2.pdf). So erhält die Stadt Duisburg beispielsweise wegen ihrer schlechten Finanzausstattung zusätzlich $49 €$ pro Einwohner, reiche Städte wie Düsseldorf bekommen nichts.

11 Die Mittel wurden regional nach den Regierungsbezirken zu $75 \%$ nach Einwohnerzahl und $25 \%$ nach Finanzkraft verteilt und können für verschiedene Aufgabenbereiche von den Kommunen beantragt werden. Finanzschwache Kommunen sollen besonders berücksichtigt werden. Ihr Eigenanteil kann auf bis zu $10 \%$ gesenkt werden (http://www.bayern.de/Konjunkturpaket-II-.2040. 10221877/index.htm).

12 Dazu dürfte auch die Vereinfachung und Lockerung des Vergaberechtes beitragen.

13 Dies bedeutet, dass die Leistung erbracht ist und nach Kontrolle zur Zahlung angewiesen wurde.
} 
steigertes Bauvolumen bearbeiten. Auch ist ungeklärt, ob das Ausbaugewerbe, das besonders für die energetische Sanierung der Gebäude zuständig ist, in der Lage sein wird, das gesteigerte Volumen ohne Kapazitätsengpässe abzuarbeiten. Andernfalls wäre die Folge eine übermäßige Preissteigerung am Bau, womit den Kommunen nicht gedient wäre. Schließlich ist bei der Beurteilung auch zu beachten, dass die Finanzierung des Programms durch Kredite geschieht, die von der folgenden Generation zurückgezahlt werden muss.

\section{Schlussbemerkung}

Das Konjunkturprogramm II der Bundesregierung dürfte seine Wirkung auf die kommunale Investitionstätigkeit nicht verfehlen. Die kommunalen Spitzenverbände prognostizieren für das Jahr 2009 einen (nominalen) Investitionszuwachs von knapp $16 \%$ sowohl in den alten wie in den neuen Bundesländern (Bundesverband der kommunalen Spitzenverbände 2009). Ein weiterer Teil des Mittelzuflusses wird wahrscheinlich für die bauliche Unterhaltung ausgegeben werden. ${ }^{14}$ Entsprechend der Konstruktion des Programms dürfte sich dieser Zuwachs vor allem im Schulbereich zeigen, wodurch eine jahrelange Forderung der Bildungspolitiker nach besseren Schulbauten eingelöst wird.

Sonderausgabenprogramme stellen aber letztlich keine dauerhafte Lösung der Deckung des kommunalen Investitionsbedarfs und der Verhinderung von Investitionsstaus dar. Es muss auf längere Sicht gelingen, die für das Funktionieren der kommunalen Infrastruktur notwendigen Mittel aus den normalen Haushalten zu generieren. Dazu bedarf es einer besseren Ausstattung mit Mitteln, einer besseren Mittelverwendung, aber auch der gesteigerten Wahrnehmung bei Bürgern und der Politik: Die Erneuerung der Infrastruktur braucht eine höhere Priorität in den politischen Entscheidungen.

\footnotetext{
14 Die Mittel des Zukunftsinvestitionsgesetzes werden als investive Maßnahmen bezeichnet und so auch im Bundeshaushalt verbucht (schon um sie mit Krediten finanzieren zu können), ein Teil wird aber auch für bauliche Unterhaltung verwendet werden, schon um die Mittel noch 2009 ausgeben zu können.
}

\section{LITERATUR}

Anton, S./Diemert, D. (2008): Gemeindefinanzbericht 2008, in: Der Städtetag 5, S. $19 \mathrm{ff}$.

Barnes, G./Langworthy, P. (2003): The per-mile operating costs of automobiles and trucks, Minnesota

Branham, D. (2002): The Wise Man Builds His House Upon the Rock: The Effects of Inadequate School Infrastructure on Student Performance, Houston

Díaz Muriel, C. (2007a): Comparative price levels for construction in 33 European countries for 2005, in: Eurostat Statistics in focus 108

Díaz Muriel, C. (2007b): Comparative price levels for investment goods in 33 European countries for 2005, in: Eurostat Statistics in focus 109 Keller, M./Wüthrich, Ph./Wittwer, S. (2007): Staukosten des Straßenverkehrs in der Schweiz, Aktualisierung 2000/2005, Bern

Lawrence, B. K. (2003): Save a Penny, Loose a school: The real cost of deferred maintenance, Washington D. C.
Reidenbach, M. (2006): Die Sachinvestitionen der Kommunen und ihrer Unternehmen, eine Bestandsaufnahme, in: Aktuelle Information des Difu, Februar

Reidenbach, M./Bracher, T./Grabow, B./Schneider, S./Seidel-Schulze, A. (2008): Investitionsrückstand und Investitionsbedarf der Kommunen. Ausmaß, Ursachen, Folgen, Strategien, Difu - Stadt Forschung Praxis, Bd. 4

Sachverständigenrat zur Begutachtung der gesamtwirtschaftlichen Entwicklung (SVR) (Hrsg.) (2008): Die Finanzkrise meistern - Wachstumskräfte stärken, Jahresgutachten 2008/2009

Statistisches Bundesamt (2008): Volkswirtschaftliche Gesamtrechnungen, Inlandsproduktberechnung, Detaillierte Jahresergebnisse, Fachserie 18, Reihe 1.4, Wiesbaden

Sutherland, L. C./Lubman, D. (2001): The Impact of Classroom Acoustics on Scholastic Achievement, http://www.nonoise.org/quietnet/qc/ ICA2001.htm 